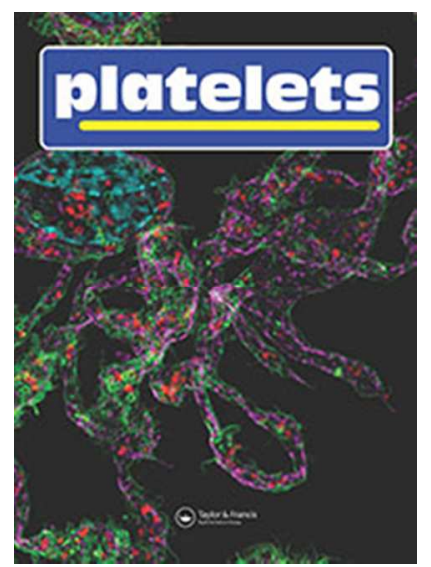

\title{
A Comprehensive Proteomics Study on Platelet Concentrates: Platelet Proteome, Storage Time and Mirasol Pathogen Reduction Technology
}

\begin{tabular}{|r|l|}
\hline Journal: & Platelets \\
\hline Manuscript ID & CPLA-2017-0249.R3 \\
\hline Manuscript Type: & Original Article \\
\hline Date Submitted by the Author: & n/a \\
\hline Complete List of Authors: & $\begin{array}{l}\text { Salunkhe, Vishal; Sanquin Research, Blood Cell Research } \\
\text { De Cuyper, Iris; Sanquin Research, Blood Cell Research } \\
\text { Papadopoulos, Petros; Hospital Clínico San Carlos, IdISSC, Hematology } \\
\text { van der Meer, Pieter; Sanquin Blood Bank, Product and Process } \\
\text { Development } \\
\text { Daal, Brunette; Sanquin Blood Bank, Product and Process Development } \\
\text { Villa-Fajardo, María; Hospital Clínico San Carlos, IdISSC, Hematology } \\
\text { de Korte, Dirk; Sanquin Blood Bank, Product and Process Development; } \\
\text { Sanquin Research, Blood Cell Research } \\
\text { van den Berg, Timo K.; Sanquin Research, Blood Cell Research } \\
\text { Gutierrez, Laura; Hospital Clínico San Carlos, IdISSC, Hematology; } \\
\text { Sanquin Research, Blood Cell Research }\end{array}$ \\
\hline Keywords: & $\begin{array}{l}\text { proteomics, platelet transfusion, platelet function tests, blood safety, } \\
\text { pathogen inactivation technologies, storage time }\end{array}$ \\
\hline
\end{tabular}

\section{SCHOLARONE \\ Manuscripts}


A Comprehensive Proteomics Study on Platelet Concentrates: Platelet Proteome, Storage Time and Mirasol Pathogen Reduction Technology

Vishal Salunkhe ${ }^{1 *}$, Iris M. De Cuyper ${ }^{1 *}$, Petros Papadopoulos ${ }^{2 *}$, Pieter F. van der Meer ${ }^{3}$, Brunette B. Daal ${ }^{3}$, María Villa-Fajardo ${ }^{2}$, Dirk de Korte ${ }^{1,3}$, Timo K. van den Berg ${ }^{1}$ and Laura Gutiérrez $^{1,2}$

${ }^{1}$ Department of Blood Cell Research, Sanquin Research and Landsteiner Laboratory, Academic Medical Centre (AMC), University of Amsterdam (UvA), Amsterdam, The Netherlands.

${ }^{2}$ Department of Hematology, Hospital Clínico San Carlos, Instituto de Investigación Sanitaria San Carlos (IdISSC), Madrid, Spain.

${ }^{3}$ Department of Product and Process Development, Sanquin Blood Bank, Amsterdam, The Netherlands.

* Equal contribution.

\section{Correspondence:}

Laura Gutiérrez, Hospital Clínico San Carlos, Dept. of Hematology, Planta Primera Sur, Room \#1578, C/Profesor Martín Lagos S/N, 28040 Madrid (Spain). Tel.: +34-91-3303861. Email: lgutierrezg@salud.madrid.org.

\section{Short Title (max 50 characters):}

Platelet Proteomics, Storage Time and Mirasol PRT (49 characters)

Abstract (max 400 words): 309 words

Manuscript: 3998 words

References: 61

Figures: 6

Tables: 1

Supporting Files: 2 Figures and 4 Tables

\section{Keywords}

Platelet Transfusion, Platelet Function Tests, Proteomics, Blood Safety, Pathogen Inactivation Technologies, Storage Time 


\begin{abstract}
Platelet concentrates (PCs) represent a blood transfusion product with a major concern for safety as their storage temperature $\left(20-24^{\circ} \mathrm{C}\right)$ allows bacterial growth, and their maximum storage time period (less than a week) precludes complete microbiological testing. Pathogen inactivation technologies (PITs) provide an additional layer of safety to the blood transfusion products from known and unknown pathogens (such as bacteria, viruses and parasites). In this context, PITs (such as Mirasol Pathogen Reduction Technology -PRT-) have been developed and are implemented in many countries. However, several studies have shown in vitro that Mirasol PRT induces a certain level of platelet shape change, hyperactivation, basal degranulation and increased oxidative damage during storage. It has been suggested that Mirasol PRT might accelerate what has been described as the platelet storage lesion (PSL), but supportive molecular signatures have not been obtained. We aimed at dissecting the influence of both variables, i.e. Mirasol PRT and storage time, at the proteome level. We present comprehensive proteomics data analysis of Control PCs and PCs treated with Mirasol PRT at storage days 1, 2, 6 and 8 . Our workflow was set to perform proteomics analysis using a gel-free and label-free quantification (LFQ) approach. Semi-quantification was based on LFQ signal intensities of identified proteins using MaxQuant/Perseus software platform. Data are available via ProteomeXchange with identifier PXD008119. We identified marginal differences between Mirasol PRT and control PCs during storage. However, those significant changes at the proteome level were specifically related to the functional aspects previously described to affect platelets upon Mirasol PRT, and in addition, the effect of Mirasol PRT on the platelet proteome appeared not to be exclusively due to an accelerated or enhanced PSL. In summary, semi-quantitative proteomics allows to discern between proteome changes due to Mirasol PRT or PSL, and proves to be a methodology suitable to phenotype platelets in an unbiased manner, in various physiological contexts.
\end{abstract}




\section{INTRODUCTION}

Transfusion transmitted infections (TTIs) pose a threat in transfusion practice as there are still infections for which routine tests are not available or cannot be performed at each hospital or blood bank, i.e. Chikungunya virus, Dengue virus, Human Parvovirus B19, Toxoplasma or Babesia [1, 2]. Although bacterial screening of blood products is mandatory in many countries, studies have shown that bacterial infections represent a major threat to transfusion products (especially platelets) compared to viral infections [1-4]. Since platelet concentrates (PCs) are stored at room temperature $\left(20-24^{\circ} \mathrm{C}\right)$ approximately for 1 week, they are at risk of contamination because this temperature allows the growth of bacteria that might be present and the storage period precludes complete microbiological testing. It has been shown that bacteria from the skin flora at the phlebotomy site are present in $70 \%$ of the transfusionrelated sepsis cases $[5,6]$. Therefore, in order to minimize the infection risk of transfusion products, Pathogen Inactivation Technologies (PITs) were developed [7, 8].

As of November 2017, Mirasol PRT (Terumo BCT, USA) PCs treatment has been approved and is in routine use in 22 countries (Susanne Marschner, personal communication). It consists of the addition to PCs of riboflavin -a naturally occurring photosensitizer that binds to nucleic acids (DNA or RNA)- followed by UV illumination (265-370 nm), which causes nucleic acid single strand breaks that cannot be repaired in pathogens [4, 9-12]. Mirasol PRT can reduce the pathogen-load including a wide range of viruses and bacteria by 4 to $6-\log$ [13-15]. Additionally, Mirasol PRT prevents leukocyte proliferation and cytokine production, counteracting potential risks of alloimmunization [16-20].

The positive aspect of Mirasol PRT is that riboflavin and its metabolic by-products do not need to be removed after UV illumination because they are naturally occurring and FDAapproved [21]. A randomized clinical trial conducted in France demonstrated that transfusion 
of Mirasol PRT PCs is not associated with adverse reactions in patients compared to Control PCs, supporting their safety. However, although the post-transfusion platelet corrected count increment $(\mathrm{CCI})$ values were above the required minimum threshold, they were lower in the group transfused with Mirasol PRT PCs [22]. Several in vitro studies showed that some quality parameters were unaffected until day 5 of storage in Mirasol PRT PCs compared to Control PCs [23-27]. Our previous studies showed that Mirasol platelets undergo spontaneous aggregation in the absence of agonist (hyperactivation), due to basal continuous platelet degranulation induced by Mirasol PRT, and that this effect was more severe late upon storage [27]. Additionally, it has been shown that Mirasol PRT enhances GPIb $\alpha$ shedding and desialylation of platelet surface proteins, affects platelet adhesion and induces shape changes to platelets late upon storage [28, 29].

In order to gain further insight on the performance of Mirasol treated PCs after transfusion, a phase III randomized clinical trial - Pathogen Reduction Evaluation \& Predictive Analytical Rating Score (PREPAReS; Sanquin Blood bank, The Netherlands), in collaboration with Terumo BCT, with bleeding as a primary endpoint, has been conducted (NTR2106, http://www.trialregister.nl) [30].

Various blood components (plasma, platelets, RBCs) have been studied using proteomic approaches lately [31]. Since platelets are anucleated entities containing a proteome of the order of thousand [31], it is feasible to measure qualitative and (ideally) quantitative changes in PCs upon storage and/or Mirasol PRT. In this study we have used an unbiased, label-free quantification (LFQ) proteomics approach that allows the comparison of global trends in the proteomes of the studied groups. With this technique we aimed at gaining insight on the effects of Mirasol PRT and storage on the platelet proteome at the qualitative and semiquantitative level. This analysis allowed us to relate the limited identified proteomic changes 
to the in vitro effects observed by us and others, and highlights the usefulness of proteomics to comprehend platelet biology in other physiological and pathophysiological contexts. 


\section{METHODS}

\section{Experimental design}

PCs were produced and stored at Sanquin Dutch Blood Bank according to national standards, meeting the requirements for Mirasol PRT. 105 healthy donors participated after giving informed consent. The study was approved by our institute medical ethics committee in accordance with the 1964 Declaration of Helsinki.

Per experiment, and as previously described [27], six buffy coat derived PCs were pooled at day 1 after blood donation and processing, and split back in six equal units. Three of these units were left untreated while the other three were treated with Mirasol PRT (Terumo BCT, Zaventem, Belgium). We collected samples from day 1 PCs before treatment, and from a single Control and a single Mirasol PRT unit at day 2, 6 and 8 of storage. Platelets and supernatants were separated by centrifugation (4000 rpm 5 minutes), and the supernatant fraction was collected after an additional centrifugation step (10000 rpm 1 minute). Platelet pellets and PC supernatants were snap frozen and stored at $-80^{\circ} \mathrm{C}$. The experiment was replicated several times, and we gathered 3 Control and 4 Mirasol PRT sets, for which different donors were employed. Freshly isolated platelets and plasma were obtained by centrifugation from citrate-anticoagulated blood sampled from three independent donors.

\section{Protein extraction and processing for mass spectrometry acquisition}

Platelets were lysed in $8 \mathrm{M}$ urea (ThermoFisher Scientific, Waltham, MA, USA) in 100mM TRIS-HCl pH 8 (ThermoFisher Scientific). $5 \mu \mathrm{g}$ protein were used for further in-solution processing. Disulfide bonds were reduced with 10mM DTT (ThermoFisher Scientific) 60 minutes at $20^{\circ} \mathrm{C}$, alkylated with $55 \mathrm{mM}$ iodocetamide (ThermoFisher Scientific) 45 minutes at $20^{\circ} \mathrm{C}$. Samples were digested 16 hours at $20^{\circ} \mathrm{C}$ with MS-grade trypsin (Promega, Fitchburg, WI, USA) in a 1:20 trypsin:protein ratio. Peptides were desalted and concentrated using 3M 
Empore-C18 StageTips (Sigma-Aldrich, St. Louis, MO, USA) as described [32] and eluted with $0.5 \%$ acetic acid (Sigma-Aldrich), $80 \%$ acetonitrile (Biosolve Chimie, Dieuze, France). Sample volume was reduced by SpeedVac and supplemented with $2 \%$ acetonitrile, $0.1 \%$ TFA (ThermoFisher Scientific) to a final volume of $5 \mu$, of which $3 \mu$ were injected for MS acquisition. Peptides were separated by nanoscale C18 reverse phase chromatography coupled on line to an Orbitrap Fusion Tribrid mass spectrometer (ThermoFisher Scientific) via a nanoelectrospray ion source (Nanospray Flex Ion Source, ThermoFisher Scientific), following standard procedures and settings, and acquired with Xcalibur software.

\section{Proteomics data analysis}

The mass spectrometry data were analysed with MaxQuant software version 1.5.5.1 using default settings and Label Free Quantification (LFQ), searching against the UniProt KB human taxonomy database [33-35]. The output Protein Groups table generated was used for further analysis with Perseus software version 1.5.5.3. A $\log 2$ transformation was applied to all LFQ intensity values. Data was filtered so that at least 1 group had at least 3 valid values. Missing values were imputed using normal distribution. All T-Tests, with multiple testing correction, and Multiple-Sample Tests were performed setting FDR at 5\% (BenjaminiHochberg) and $\mathrm{S}_{0}=0$ [36]. Soft clustering of proteins was done using K-means. The mass spectrometry proteomics data have been deposited to the ProteomeXchange Consortium via the PRIDE partner repository with the dataset identifier PXD008119 [37-39]. GO-Term searches were performed using STRING web-based software platform, version 9.1 (http://string-db.org/) [40, 41].

Flow cytometry-based platelet aggregation assay (FCA)

Flow cytometry-based platelet aggregation assay was performed as described [42]. The agonists used were phorbol myristate acetate (PMA-100 ng/mL), convulxin $(2 \mu \mathrm{g} / \mathrm{mL}$ ), collagen $(10 \mu \mathrm{g} / \mathrm{mL})$ and ristocetin $(1.5 \mathrm{mg} / \mathrm{mL})$. Aggregation was measured by flow 
cytometry (LSRII+HTS, BD Biosciences, San José, CA, USA) through a time-series per agonist, and data was analysed with FlowJo software (Version 9.2, Tree Star Inc., Ashland, OR, USA). The area under the curve (AUC) of each reaction was used as aggregation capacity variable. Student's T-test analyses were performed in order to identify significant differences.

\section{Western blot analysis}

Platelet lysates (RIPA), or PC supernatants depleted of albumin/IgG (Pierce ${ }^{\mathrm{TM}}$ Albumin/IgG Removal Kit, \#89875, ThermoFisher Scientific) were subject to 1D-SDS-PAGE and immunoblotting was performed. We used as primary antibodies anti-EMILIN1 (ab56936, Abcam, Cambridge, UK), anti-NID1 (AF2570), anti-NID2 (AF3385) and anti-THBS1 (AF3074) from Bio-Techne (Minneapolis, MN, USA); anti-GP5 (sc-271662), anti-APOH (sc-515677) and anti-MPL (H300, sc-15403) from Santa Cruz Biotechnology (Dallas, TX, USA); anti-GAPDH (MAB374, Darmstadt, Merck, Germany); and as secondary antibodies we used anti-mouse (\#31430) and anti-rabbit (\#31460) from ThermoFisher Scientific; antigoat (A16005, Invitrogen, Carlsbad, CA, USA); or anti-mouse (NA93IV, GE Healthcare, Little Chalfont, UK) all IgG HRP conjugated. Membranes were developed with Chemiluminescence (Amersham, Little Chalfont, UK). Gels were stained with Imperial ${ }^{\mathrm{TM}}$ Protein Stain \#24615 or with Pierce ${ }^{\mathrm{TM}}$ Glycoprotein Staining Kit \#24562 (ThermoFisher Scientific). 


\section{RESULTS}

Mirasol PRT induces functional changes, i.e. hyperactivation and basal degranulation $[23,25$, 27, 43]. It has been suggested that Mirasol PRT accelerates the platelet storage lesion (PSL) on PCs. To gain insight into this functional phenomenon, and complementing our previous observations [27], we took advantage of a flow-cytometry based platelet aggregation assay that allows the study of the contribution of single receptors to the early steps of platelet aggregation, depending on the agonist applied, to compare platelet responses between Control and Mirasol PRT PCs (Figure 1) [42]. We used as agonists PMA (activating $\alpha 2 b \beta 3$ ), ristocetin (activating vWF-Receptor), convulxin (activating GP6) and collagen (activating both GP6 and $\alpha 2 \beta 1$ ), and freshly isolated platelets as a reference. As known [44-46], Control PCs platelets lose aggregation capacity upon storage, and our assay shows that it is $\alpha 2 \beta 1$ and, to a lesser extent, vWF-R- and GP6-mediated responses that are affected. Mirasol PRT platelets displayed more severely reduced vWF-R- and GP6-mediated responses, while the $\alpha 2 \beta 1$-mediated response was equally affected compared to Control PC platelets. $\alpha 2 \mathrm{~b} \beta 3$ mediated responses are generally preserved in both groups. This data suggests that the functional capacity loss observed in Control and Mirasol PRT PCs upon storage is characteristic, supporting the notion that the processes affected by either Mirasol PRT or PSL are distinct.

\section{A proteomic approach to unravel differences induced by Mirasol PRT and/or storage}

We questioned ourselves whether we could distinguish between Mirasol PRT and PSLrelated defects in an unbiased manner and therefore, we aimed at analysing alterations in the platelet proteome of Mirasol PRT and Control PCs during storage (Figure 2A-D). Prior mass spectrometry acquisition, platelet protein extracts prepared from fresh platelets, day 1 PCs before treatment, and Control or Mirasol PRT replicate PCs at different time points during storage (i.e. day 2, 6 and 8) were subject to in-solution trypsin digestion (as opposed to in-gel 
digestion), in order to reduce the processing steps of the sample, while still gaining deep protein coverage.

Identification and quantitation of proteins was done with label-free quantification (LFQ) using MaxQuant and Perseus software platforms (PRIDE accession number PXD008119). We identified 3445 protein groups across samples and 40537 corresponding peptide sequences. We next filtered identified proteins from 3445 to 2505 by removing potential contaminants (with the exception of THBS1 and TPM2, which are present in platelets), only identified by site, reverse sequences and duplicate entries (i.e. protein groups). Principal Component Analysis (PCA) of all samples analysed shows three populations: Control PCs together with fresh platelets, Mirasol PRT, and, as an isolated population, day 1 PCs (Figure 2E).

\section{Comparison of the proteome of Control and Mirasol PRT PCs through storage}

We compared the Control and Mirasol PRT PCs proteomes at day 2, 6 and 8 of storage, in order to identify potential proteomic differences (Table S1). Importantly, PCA and Pearson correlation analysis of replicates within the Control and Mirasol PRT groups showed that Control samples correlated strongly with each other, while Mirasol PRT samples displayed more heterogeneity, i.e. some of the Mirasol PRT samples cluster together with Control samples, and do not correlate strongly within the Mirasol PRT group (Figure 3A-B). This suggests that the effect of Mirasol PRT on the PC proteome ranges, most probably on a donor-dependent manner.

Unsupervised hierarchical clustering of Control and Mirasol PRT PCs averaged per time point generated distinct clusters, separating Mirasol PRT from Control PCs (Figure 3C). The first cluster included Control PCs at day 2, the second included Control PCs at days 6 and 8, the third included Mirasol PRT PCs at day 2 and the fourth comprised Mirasol PRT PCs at 
days 6 and 8. Both Control and Mirasol PRT PCs samples at day 2 separated from the rest. The clustering of Control or Mirasol PRT PCs at days 6 and 8 suggests respective common proteomic changes late during storage. However, based on Pearson index, day 6-8 Control or Mirasol PRT PCs do not correlate strongly amongst each other, suggesting distinct changes due to Mirasol PRT (Figure 3D).

\section{Proteome alterations due to Mirasol PRT are minimal but increase with storage time}

We performed T-tests in order to identify differences at the proteome level amongst treatments at each day of storage, however, we could not identify any (Table S1). However, multiple-sample test analysis led to the identification of a total of 20 proteins that were significantly differentially expressed (DE) between Mirasol PRT and Control PC samples (Table 1 and Table S1). Unsupervised hierarchical clustering was performed and is shown in Figure 4A. Interestingly, the majority of downregulated DE proteins are contained in $\alpha$ granules [47] and are related to platelet functions such as adhesion, activation and degranulation by GO-Term search (Figure 4, Table S2 and Figure S1). Furthermore, upregulated DE proteins locate mainly in mitochondria and plasma membrane. This data supports the notion that Mirasol PRT induces basal platelet degranulation (as downregulated proteins, which locate to $\alpha$-granules, are probably secreted). Upregulated (or enriched proteins that are not secreted thereof) locate to mitochondria and membrane domains, corroborating previous findings that Mirasol PRT does not alter mitochondria integrity in PCs $[23,27]$.

We analysed by Western blotting (Figure 5A) the expression of some of the DE proteins and unaltered ones in platelet lysates. We confirmed downregulation of EMILIN1, GP5, NID1, NID2 and THBS1, some of them being visibly reduced by day 8 , others affected already by day 6. We confirmed overexpression of APOH, while MPL and GAPDH were barely 
affected in Mirasol PRT compared to Control platelets. Coomassie staining shows that the protein concentration is homogeneous. Curiously, GP5 is also downregulated in Control PCs, however, the downregulation is more severe in Mirasol PRT. This correlates with the dynamics of vWF-R-mediated responses, which are affected in both groups, although more severely in Mirasol PRT platelets, upon storage (Figure 1) [29].

In order to prove that downregulation of proteins in Mirasol PRT platelet proteomes is due to degranulation, we analysed their expression in the supernatants of respective samples (Figure 5B). Data shows presence of EMILIN1 in Mirasol PRT supernatants, while it is undetectable in Control PCs and fresh plasma sample. Regarding NID1 and NID2, they are barely detectable in fresh plasma samples, and they are detectable in Control supernatants, however the amount is higher -and earlier during storage- in Mirasol PRT supernatants in the case of NID2. Interestingly, the amount of protease-cleaved NID1 (arrowheads) is higher in Mirasol PRT supernatants, suggesting active proteolysis, occurring at a higher extent than in Control PCs. Coomassie staining shows that the amount of protein present in supernatants is higher at day 8 in both Control and Mirasol PRT samples, and arrowheads display bands altered on sample protein patterns. Interestingly, Glycoprotein staining reveals extra bands in Mirasol PRT supernatants early upon storage, bands that are not detectable in fresh plasma or Control supernatants (weak bands are seen only at day 8). This supports the notions that there is active Mirasol PRT-dependent protein release from platelets, and that there might be active proteolysis, as consequence of degranulation-dependent release of platelet proteases.

\section{Comparison of the proteome of fresh platelets and Control PCs through storage}

The recommended shelf life of PCs, ranging from 3 to 7 days, is still a matter of discernment amongst different countries, where transfusion policies differ due to both the risk of bacterial contamination and the acknowledged platelet storage lesion (PSL) [48, 49]. PSL is associated 
with reduced in vivo platelet recovery, survival and haemostatic activity after transfusion [50]. To identify protein changes due to storage, the proteome of freshly isolated platelets was compared with the proteome of platelets from PCs at storage days 1, 2, 6 and 8 (Figure 2 and Table S3).

PCA and Pearson correlation analysis showed that in general, fresh (D0) platelet proteome differs from stored Control PCs (Figure 6A-B), however, day 1 PCs samples separate clearly from further stored or fresh platelet samples. Unsupervised hierarchical clustering generated distinct clusters (Figure 6C) and showed a unique proteome from each sample. Consequently, these samples did not correlate with each other, although fresh platelets tend to pair with day 2, and day 6 with day 8, leaving day 1 PCs platelets isolated (Figure 6D). Our explanation for this is that day $1 \mathrm{PC}$ platelet proteomes might be skewed due to a percentage of old, hyperreactive or apoptotic platelets that are "stressed and visible" due to recent sampling and processing.

None of the identified proteins were significantly DE taking either fresh or day 1 stored platelets as reference (with isolated exceptions) and we also did not identify differentially expressed proteins employing multiple-sample test analysis (Table S3).

However, we wanted to evaluate potential protein level dynamics influenced by storage that could explain the functional differences observed between Mirasol PRT and Control PCs or those dependent on storage. Therefore, we performed cluster analysis of protein expression dynamics during platelet storage (days 2, 6 and 8), (Figure S2 and Table S4). Particularly interesting were clusters $4,6,8$ and 9 , in which respective protein groups undergo downregulation at the beginning or end of storage (being clusters 1,2 and 7 their mirror responsive clusters respectively). GO-Term analysis from clusters 1-9 is provided in Table S4. In general, there are a number of proteins in these clusters that participate in vesicular 
transport and metabolic pathways, which do not correlate to the GO-Term enrichment derived from the DE proteins induced by Mirasol PRT. In addition, the expression tendencies are not shared when comparing the effects of Mirasol PRT or storage, i.e. not all downregulated proteins due to Mirasol PRT are downregulated in Control platelets through storage.

Summarizing, storage does not critically affect the platelet proteome, although there were protein expression dynamics identified through cluster analysis, which supported the lowered aggregation capacity displayed late during storage and the partial overlap with Mirasol PRTinduced proteomic changes. Furthermore, our data show that Mirasol PRT generates minimal alterations at the proteome level, however, those alterations are linked to the functional changes that have been observed (i.e. basal degranulation) and are more severe upon storage [27]. 


\section{DISCUSSION}

Several in vitro studies have reported the effect of Mirasol PRT on platelet quality, and while the conclusions on the extent of the impact of Mirasol PRT on PCs differs among studies, it is generally acknowledged that Mirasol PRT, and other PITs, do exert an effect on the treated transfusion product $[23,25,27,51-54]$. Here we applied state-of-the-art mass spectrometry technology to analyse the effect of Mirasol PRT on the platelet proteome. We have identified 20 DE proteins (out of $\sim 2500$ ) upon Mirasol PRT in comparison to Control PCs, suggesting that very few proteins are affected. Our results are in agreement with previous studies showing that Mirasol PRT affected only a handful of proteins (mainly related to the cytoskeleton and metabolism), despite the differences in methods and data analyses employed, i.e. in-solution digestion and label-free quantification (LFQ) vs in-gel digestion $[24,55]$. Such knowledge might contribute to the development of alternatives to improve PIT PCs quality parameters and maximize the efficacy of a safe transfusion product. For example, a study reported that p38MAPK was hyper-phosphorylated in Mirasol PRT platelets, and that adding a p38MAPK-specific inhibitor before treatment, resulted not only in decreased phosphorylation of its substrates such as VASP, AKT and HSP27, but also on improved platelet function and survival $[56,57]$.

Interestingly, none of these 20 proteins identified in the present study appear in earlier proteomic studies in Mirasol treated platelets, with the exception of THBS1 [24, 28, 55]. Further analysis of the MS data revealed that most of these DE proteins are components of $\alpha$ granules of platelets $[47,58]$, which support our previous findings that Mirasol PRT leads to platelet hyperactivation due to basal degranulation [27]. Platelet degranulation is accompanied by the release of proteases, which result in shedding of receptors such as GPIb $\alpha$ and platelet desialylation, as reported [29]. Identified platelet proteases ADAM10 and MMP1 
(Table S1), get reduced by Mirasol PRT -although not significantly-. Platelet released proteases and those present in plasma, might account for the enhanced cleavage of susceptible proteins, such as NID1 [59]. It has been described that NID1, a component of basal membranes involved in cell-matrix interactions, binds to fibrin and fibrinogen [60]. However, it is not known whether the increased levels of NID1 (and general released/secreted platelet cargo) or proteolytic products may alter haemostasis or influence tissue regeneration [59]. Glycoprotein and Coomassie staining of PCs supernatants revealed different patterns between Mirasol PRT compared to fresh plasma, suggesting active modification of plasma protein components. However, how these modifications might affect transfusion product performance is unknown. This aspect also adds to the current opinion that Platelet Additive Solution (PAS) might be preferred over the use of plasma for PC storage [61]. It is important to acknowledge that downregulation of proteins such as MMRN1 or EMILIN1 in the platelet proteome, is not necessarily caused by the same biological process (i.e. degranulation). Control PCs experience also downregulation of MMRN1 and EMILIN1 through storage, based on our Cluster analysis, however, we have evidence that it is not merely due to basal or active degranulation, but other processes may play a role, such as vesiculation.

We observed that upon storage, ristocetin-mediated responses were reduced, and this reduction was more severe on Mirasol PRT PCs. Ristocetin induces platelet agglutination through the GP1b-IX-V receptor complex; our proteomics data showed downregulation of GP5 upon Mirasol PRT, and cluster-based analysis shows that GP5 expression dynamics (cluster 4) display a reduction tendency in Control PCs. GP5 downregulation was observed by Western blot analysis in Control PCs, downregulation that was more severe on Mirasol PRT PCs (Figure 5). These data goes along the ristocetin-mediated response reduction in PCs, which is more severe in Mirasol PRT PCs, and recent in vitro studies that show that 
Mirasol PRT enhances GPIb $\alpha$ shedding and platelet desialylation [29]. The recentlycompleted PREPAReS trial showed minor differences in the percentage of patients with major bleeding complications after transfusion, comparing Mirasol PRT with Control PCs [30]. However, it remains to be evaluated whether those minor differences correlate with the transfusion of older vs younger PCs, i.e. the storage time.

When analysing the platelet proteome of Control PCs through storage, we could not identify DE proteins. However, K-means cluster analysis suggested proteomic dynamic changes through storage. From the 20 proteins DE upon Mirasol PRT, not all were affected or changed in the same direction, based on cluster analysis. From the downregulated DE proteins upon Mirasol PRT, only GP5, MMRN1, EMILIN1, NID2, THBS1 and TSC22D3 belong to clusters with clear expression downregulation upon storage, while NID1, MLEC, ENDOD1, TMED7 and VAT1 follow opposite kinetics. On the other hand, from the upregulated DE proteins upon Mirasol PRT, only FH, DECR1 and ALDH4A1 follow the same pattern upon storage in Control PCs. Therefore, cluster analysis revealed that the proteome profile of Mirasol PRT and storage lesion overlaps partially, although proteomic signatures are distinct.

Our study has some limitations, as we discarded protein hits with poor peptide coverage, and protein modifications or fragmentation were not studied. It has been shown that oxidative damage was induced by Mirasol and Intercept (Amotosalen) treatments on model peptides [53]. Results from this study revealed that Mirasol PRT generated more oxidation on peptides and Mirasol and Amotosalen induced different peptide modifications at different sites. In addition, the identification of low abundant proteins in platelet lysates might be an extra limitation of the current methodology, and significant differences identified depend on the stringency of the statistical analysis performed. 
In summary, we have demonstrated that Mirasol PRT impacts few proteins in platelets and these are mostly related to platelet adhesion, activation and degranulation. However, we did not find significant DE proteins between fresh and stored platelets and cluster analysis suggests that PSL and Mirasol PRT have partially overlapping but distinct signatures.

\section{ACKOWLEDGEMENTS}

We thank Eric Gouwerok, Mya Go and Richard Vlaar for technical support and Dr. Juan Carlos Rodríguez-Manzaneque for technical advice and scientific discussion. We are thankful to Dr. Susanne Marschner and Dr. Angel García for critically reviewing the manuscript. We are also thankful to Terumo BCT for supplying the illuminator and disposables at no cost.

\section{AUTHOR CONTRIBUTIONS}

VS, PvdM, DdK and LG conceived and designed the study. VS, IMDC, PP, BBD, MVF and LG performed experiments. VS, IMDC, PP and LGG analysed data. VS, PP and LG performed proteomics analysis. VS and LG wrote the paper. IMDC, PP, PvdM, DdK, and TKvdB reviewed the manuscript.

\section{DECLARATION OF INTEREST}

V.S. was supported by the Center for Translational Molecular Medicine (CTMM, www.ctmm.nl), project Innovative Coagulation Diagnostics (INCOAG, grant 01C-201), and the Dutch Heart Foundation. L.G. was partially supported by an RYC fellowship (RYC2013-12587, Ministerio de Economía y Competitividad, Spain), an I+D Excelencia 2014 project grant (SAF2014-55231-P, Ministerio de Economía y Competitividad -Spain- and Fondos Feder) and a grant by the Spanish Society of Proteomics (SeProt 2015, Spain). P.P. was partially supported by an I+D Excelencia 2014 project grant (SAF2014-55231-P, Ministerio de Economía y Competitividad -Spain- and Fondos Feder) and Funds from the Comunidad de Madrid “Atracción de Talento” (2016-T1/BMD-1051). 


\section{REFERENCES}

1. Allain, J.P., et al., Protecting the blood supply from emerging pathogens: the role of pathogen inactivation. Transfus Med Rev, 2005. 19(2): p. 110-26.

2. Allain, J.P., et al., Transfusion-transmitted infectious diseases. Biologicals, 2009. 37(2): p. 717.

3. Cable, R.G. and D.A. Leiby, Risk and prevention of transfusion-transmitted babesiosis and other tick-borne diseases. Curr Opin Hematol, 2003. 10(6): p. 405-11.

4. Salunkhe, V., et al., Development of blood transfusion product pathogen reduction treatments: a review of methods, current applications and demands. Transfus Apher Sci, 2015. 52(1): p. 19-34.

5. Brecher, M.E. and S.N. Hay, Bacterial contamination of blood components. Clin Microbiol Rev, 2005. 18(1): p. 195-204.

6. Kuehnert, M.J., et al., Transfusion-transmitted bacterial infection in the United States, 1998 through 2000. Transfusion, 2001. 41(12): p. 1493-9.

7. Hervig, T., J. Seghatchian, and T.O. Apelseth, Current debate on pathogen inactivation of platelet concentrates--to use or not to use? Transfus Apher Sci, 2010. 43(3): p. 411-4.

8. Seghatchian, J. and G. de Sousa, Pathogen-reduction systems for blood components: the current position and future trends. Transfus Apher Sci, 2006. 35(3): p. 189-96.

9. Hardwick, C.C., et al., Separation, identification and quantification of riboflavin and its photoproducts in blood products using high-performance liquid chromatography with fluorescence detection: a method to support pathogen reduction technology. Photochem Photobiol, 2004. 80(3): p. 609-15.

10. Kumar, V., et al., Riboflavin and UV-light based pathogen reduction: extent and consequence of DNA damage at the molecular level. Photochem Photobiol, 2004. 80: p. 15-21.

11. Li, J., et al., Platelet glycolytic flux increases stimulated by ultraviolet-induced stress is not the direct cause of platelet morphology and activation changes: possible implications for the role of glucose in platelet storage. Transfusion, 2005. 45(11): p. 1750-8.

12. Marschner, S. and R. Goodrich, Pathogen Reduction Technology Treatment of Platelets, Plasma and Whole Blood Using Riboflavin and UV Light. Transfus Med Hemother, 2011. 38(1): p. 8-18.

13. Keil, S.D., et al., Inactivation of viruses in platelet and plasma products using a riboflavin-andUV-based photochemical treatment. Transfusion, 2015. 55(7): p. 1736-44.

14. Keil, S.D., et al., Treatment of Platelet Products with Riboflavin and UV Light: Effectiveness Against High Titer Bacterial Contamination. J Vis Exp, 2015(102): p. e52820.

15. Ruane, P.H., et al., Photochemical inactivation of selected viruses and bacteria in platelet concentrates using riboflavin and light. Transfusion, 2004. 44(6): p. 877-85.

16. Asano, H., et al., Treatment with riboflavin and ultraviolet light prevents alloimmunization to platelet transfusions and cardiac transplants. Transplantation, 2007. 84(9): p. 1174-82.

17. Fast, L.D., et al., Mirasol PRT treatment of donor white blood cells prevents the development of xenogeneic graft-versus-host disease in Rag2-/-gamma c-/- double knockout mice. Transfusion, 2006. 46(9): p. 1553-60.

18. Fast, L.D., et al., Functional inactivation of white blood cells by Mirasol treatment. Transfusion, 2006. 46(4): p. 642-8.

19. Fast, L.D., G. DiLeone, and S. Marschner, Inactivation of human white blood cells in platelet products after pathogen reduction technology treatment in comparison to gamma irradiation. Transfusion, 2011. 51(7): p. 1397-404.

20. Marschner, S., et al., White blood cell inactivation after treatment with riboflavin and ultraviolet light. Transfusion, 2010. 50(11): p. 2489-98. 
21. Reddy, H.L., et al., Toxicity testing of a novel riboflavin-based technology for pathogen reduction and white blood cell inactivation. Transfus Med Rev, 2008. 22(2): p. 133-53.

22. Mirasol Clinical Evaluation Study, G., A randomized controlled clinical trial evaluating the performance and safety of platelets treated with MIRASOL pathogen reduction technology. Transfusion, 2010. 50(11): p. 2362-75.

23. $\mathrm{Li}, \mathrm{J}$. , et al., Evaluation of platelet mitochondria integrity after treatment with Mirasol pathogen reduction technology. Transfusion, 2005. 45(6): p. 920-6.

24. Marrocco, C., et al., Proteomic analysis of platelets treated with gamma irradiation versus a commercial photochemical pathogen reduction technology. Transfusion, 2013. 53(8): p. 1808-20.

25. Perez-Pujol, S., et al., Effects of a new pathogen-reduction technology (Mirasol PRT) on functional aspects of platelet concentrates. Transfusion, 2005. 45(6): p. 911-9.

26. Picker, S.M., A. Steisel, and B.S. Gathof, Effects of Mirasol PRT treatment on storage lesion development in plasma-stored apheresis-derived platelets compared to untreated and irradiated units. Transfusion, 2008. 48(8): p. 1685-92.

27. Zeddies, S., et al., Pathogen reduction treatment using riboflavin and ultraviolet light impairs platelet reactivity toward specific agonists in vitro. Transfusion, 2014. 54(9): p. 2292-300.

28. Prudent, M., et al., Proteome changes in platelets after pathogen inactivation--an interlaboratory consensus. Transfus Med Rev, 2014. 28(2): p. 72-83.

29. Rijkers, M., et al., Evaluation of the role of the GPIb-IX-V receptor complex in development of the platelet storage lesion. Vox Sang, 2016. 111(3): p. 247-256.

30. Van Der Meer, P.F., et al.,: Hemostatic Efficacy of Pathogen-Inactivated Buffy Coat-Derived Platelet Concentrates in Hemato-Oncological Patients: Outcomes of the Prepares Trial. Blood, 2017. 130 (Suppl 1).

31. Burkhart, J.M., et al., The first comprehensive and quantitative analysis of human platelet protein composition allows the comparative analysis of structural and functional pathways. Blood, 2012. 120(15): p. e73-82.

32. Yu, Y.S., M.; Pieper, R., A spinnable and automatable StageTip for high throughput peptide desalting and proteomics. Protocol Exchange, 2014. doi:10.1038/protex.2014.033.

33. Cox, J. and M. Mann, MaxQuant enables high peptide identification rates, individualized p.p.b.-range mass accuracies and proteome-wide protein quantification. Nat Biotechnol, 2008. 26(12): p. 1367-72.

34. Cox, J., et al., Andromeda: a peptide search engine integrated into the MaxQuant environment. J Proteome Res, 2011. 10(4): p. 1794-805.

35. Luber, C.A., et al., Quantitative proteomics reveals subset-specific viral recognition in dendritic cells. Immunity, 2010. 32(2): p. 279-89.

36. Tusher, V.G., R. Tibshirani, and G. Chu, Significance analysis of microarrays applied to the ionizing radiation response. Proc Natl Acad Sci U S A, 2001. 98(9): p. 5116-21.

37. Deutsch, E.W., et al., The ProteomeXchange consortium in 2017: supporting the cultural change in proteomics public data deposition. Nucleic Acids Res, 2017. 45(D1): p. D1100D1106.

38. Perez-Riverol, Y., et al., PRIDE Inspector Toolsuite: Moving Toward a Universal Visualization Tool for Proteomics Data Standard Formats and Quality Assessment of ProteomeXchange Datasets. Mol Cell Proteomics, 2016. 15(1): p. 305-17.

39. Vizcaino, J.A., et al., 2016 update of the PRIDE database and its related tools. Nucleic Acids Res, 2016. 44(D1): p. D447-56.

40. Franceschini, A., et al., STRING v9.1: protein-protein interaction networks, with increased coverage and integration. Nucleic Acids Res, 2013. 41(Database issue): p. D808-15.

41. Jensen, L.J., et al., STRING 8--a global view on proteins and their functional interactions in 630 organisms. Nucleic Acids Res, 2009. 37(Database issue): p. D412-6. 
42. De Cuyper, I.M., et al., A novel flow cytometry-based platelet aggregation assay. Blood, 2013. 121(10): p. e70-80.

43. Johnson, L. and D. Marks, Treatment of Platelet Concentrates with the Mirasol Pathogen Inactivation System Modulates Platelet Oxidative Stress and NF-kappaB Activation. Transfus Med Hemother, 2015. 42(3): p. 167-73.

44. Leitner, G.C., et al., Additive solutions differentially affect metabolic and functional parameters of platelet concentrates. Vox Sang, 2016. 110(1): p. 20-6.

45. Ponschab, M., et al., Platelet function in reconstituted whole blood variants: An observational study over 5 days of storage time. J Trauma Acute Care Surg, 2015. 79(5): p. 797-804.

46. Shams Hakimi, C., et al., In vitro assessment of platelet concentrates with multiple electrode aggregometry. Platelets, 2015. 26(2): p. 132-7.

47. Maynard, D.M., et al., The alpha-granule proteome: novel proteins in normal and ghost granules in gray platelet syndrome. J Thromb Haemost, 2010. 8(8): p. 1786-96.

48. Seghatchian, J. and P. Krailadsiri, The platelet storage lesion. Transfus Med Rev, 1997. 11(2): p. 130-44.

49. Winkler, A.M., et al., Effects of storage duration and volume on the quality of leukoreduced apheresis-derived platelets: implications for pediatric transfusion medicine. Transfusion, 2010. 50(10): p. 2193-8.

50. Rinalducci, S. and L. Zolla, Biochemistry of storage lesions of red cell and platelet concentrates: A continuous fight implying oxidative/nitrosative/phosphorylative stress and signaling. Transfus Apher Sci, 2015. 52(3): p. 262-9.

51. Galan, A.M., et al., Impact of pathogen reduction technology and storage in platelet additive solutions on platelet function. Transfusion, 2011. 51(4): p. 808-15.

52. Ostrowski, S.R., et al., Hemostatic function of buffy coat platelets in additive solution treated with pathogen reduction technology. Transfusion, 2011. 51(2): p. 344-56.

53. Prudent, M., et al., LC-MS/MS analysis and comparison of oxidative damages on peptides induced by pathogen reduction technologies for platelets. J Am Soc Mass Spectrom, 2014. 25(4): p. 651-61.

54. Reikvam, H., et al., The Mirasol Pathogen Reduction Technology system and quality of platelets stored in platelet additive solution. Blood Transfus, 2010. 8(3): p. 186-92.

55. Schubert, P., et al., Riboflavin and ultraviolet light treatment potentiates vasodilatorstimulated phosphoprotein Ser-239 phosphorylation in platelet concentrates during storage. Transfusion, 2012. 52(2): p. 397-408.

56. Chen, Z., et al., p38MAPK is involved in apoptosis development in apheresis platelet concentrates after riboflavin and ultraviolet light treatment. Transfusion, 2015. 55(4): p. 84857.

57. Schubert, P., et al., Riboflavin and ultraviolet light treatment of platelets triggers p38MAPK signaling: inhibition significantly improves in vitro platelet quality after pathogen reduction treatment. Transfusion, 2013. 53(12): p. 3164-73.

58. Blair, P. and R. Flaumenhaft, Platelet alpha-granules: basic biology and clinical correlates. Blood Rev, 2009. 23(4): p. 177-89.

59. Martino-Echarri, E., et al., Contribution of ADAMTS1 as a tumor suppressor gene in human breast carcinoma. Linking its tumor inhibitory properties to its proteolytic activity on nidogen-1 and nidogen-2. Int J Cancer, 2013. 133(10): p. 2315-24.

60. Wu, C. and A.E. Chung, Potential role of entactin in hemostasis. Specific interaction of entactin with fibrinogen A alpha and B beta chains. J Biol Chem, 1991. 266(28): p. 18802-7.

61. van der Meer, P.F., PAS or plasma for storage of platelets? A concise review. Transfus Med, 2016. 26(5): p. 339-342. 


\section{FIGURE LEGENDS}

Figure 1. Flow cytometry-based PLT aggregation assay. Aggregation of PLTs from Control and Mirasol PRT PCs at day 2, day 6, and day 8 of storage upon addition of the agonists PMA, collagen, Convulxin, and Ristocetin over an incubation period of 5 minutes is depicted. Aggregation was measured by flow cytometry as percentage of double-colored events through a time-series course, and the Area Under the Curve (AUC) was calculated. Mean and SEM of three independent experiments are shown. D0 are fresh platelets used as reference. ${ }^{*} \mathrm{p}<0.05, * * \mathrm{p}<0.01, * * * \mathrm{p}<0.001$. When the asterisk is blue, the comparison was made with D0 fresh reference platelets. When the asterisk is grey, comparison has been made with the day Control platelet samples.

\section{Figure 2. Schematic overview of the sample preparation and proteomics workflow. (A)} Platelet concentrate (PC) preparation is done using a buffy coat-based differential centrifugation method. 1 PC unit is made of 5 units of donated whole blood (WB). RBCs, red blood cells; PLTs, platelets. (B) Experimental setup for Mirasol PRT and Control PC sampling. On each experiment, 6 PCs were pooled together and divided again into 6 units. From these, 3 units were Mirasol PRT treated and the other 3 were left untreated (Control PCs). Samples for platelet function and protein lysate preparation were collected from individual units at day 2,6 and 8. (C) Fresh platelets were isolated by differential centrifugation, and were subsequently used for functional analysis and protein lysate preparation. (D) Equal amounts (5 $\mu$ g protein) from platelet lysate samples were subject to trypsin digestion (in-solution digestion), followed by Orbitrap Fusion ${ }^{\mathrm{TM}}$ Tribrid $^{\mathrm{TM}} \mathrm{MS}$ analysis. Proteins were identified using MaxQuant version 1.5.5.1 (http://www.maxquant.org) coupled to Andromeda search engine. Subsequent label-free quantification bioinformatic analysis was performed in Perseus software version 1.5.5.3 
(http://www.perseusframework.org). (E) Principal Component Analysis of all files included in the study. D0 are fresh platelets.

Figure 3. Proteome comparison Mirasol PRT and Control PCs during storage. (A) Principal Component Analysis of Mirasol PRT and Control PCs at days 2, 6 and 8. (B) Matrix representation of Pearson correlation values of the proteome of Mirasol PRT and Control PC platelet lysate replicates through storage. (C) Two-way unsupervised hierarchical clustering of the median protein expression values of the proteome of Control and Mirasol PRT platelet lysate samples at day 2, 6 and 8 of storage. (D) Matrix representation of Pearson correlation values of the proteomes of each averaged stored Control and Mirasol PRT platelet sample-group against the others.

Figure 4. Proteins differentially expressed in Mirasol PCs are minimal, and locate to alpha-granules. (A) Two-way unsupervised hierarchical clustering of the normalized expression values of all 20 significant proteins was performed. Control and Mirasol PRT PC sample replicates were clustered. (B) Graphical representation of the DE proteins indicating their assigned cellular compartment. Most of the downregulated DE proteins are located to alpha-granules, while most of the upregulated DE proteins are located to mitochondria and plasma membrane.

\section{Figure 5. Western blot analysis of DE proteins in platelet lysates and PC supernatants.}

(A) Western blot analysis of platelet lysates: the figure panel displays representative Western blot analyses of EMILIN1, GP5, NID1, NID2 and THBS1 (downregulated, within blue box); APOH (upregulated, within pink box); and MPL and GAPDH expression in Control and Mirasol PRT platelets through storage. The lysate volume corresponding to the same number of platelets was loaded. Coomassie staining is provided as loading control, including lysate from fresh platelets (D0). (B) Western blot analysis of PC supernatants: the figure panel 
displays representative Western blot analyses of EMILIN1, NID1, NID2 and THBS1 in Control and Mirasol PRT PC supernatants through storage. D0 in this panel is plasma from fresh blood samples. The same volume of IgG/albumin depleted supernatants was loaded. Glycoprotein and Coomassie stainings are provided.

\section{Figure 6. Proteome comparison of fresh (D0) platelets and Control PCs during storage.}

(A) Principal Component Analysis of Control PCs at days 1, 2, 6 and 8. D0 are fresh platelets. (B) Matrix representation of Pearson correlation values of the proteome of stored Control and fresh (D0) platelet lysate replicates. (C) Two-way unsupervised hierarchical clustering of the median protein expression values of the proteome of fresh (D0) and stored platelet samples at day 1, 2, 6 and 8 through storage. (D) Matrix representation of Pearson correlation values of the proteomes of each averaged stored Control and fresh platelet sample-group against the others.

\section{Table 1: Significant Protein Changes after Multiple Sample Test of Control vs Mirasol} PC Lysates through Storage. Indicated are the Log2 LFQ fold ratios Mirasol vs Control at days 2, 6 and 8 of Storage, Multiple Sample test ANOVA FDR, and regulation of expression Mirasol vs Control. LFQ = Label Free Quantification; FDR = false discovery rate; $\mathrm{C}=$ Control; $\mathrm{M}=$ Mirasol, UP = upregulated; DOWN = downregulated. 


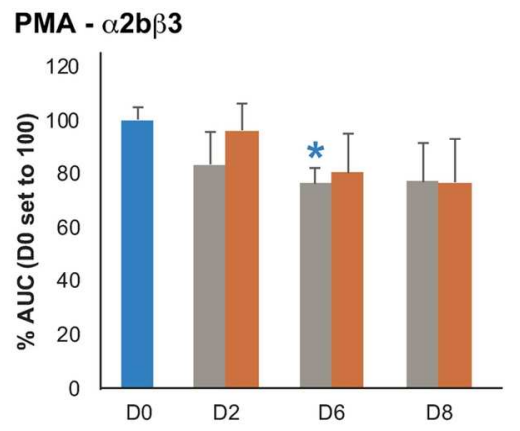

\section{Ristocetin - vWF R}

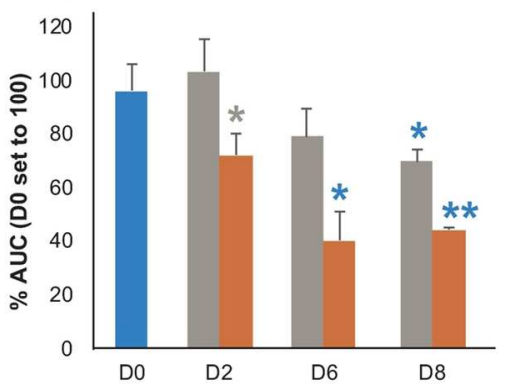

Collagen - $\alpha 2 \beta 1$ and GP6

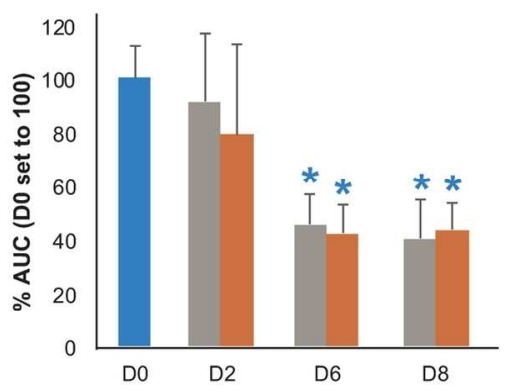

Convulxin - GP6

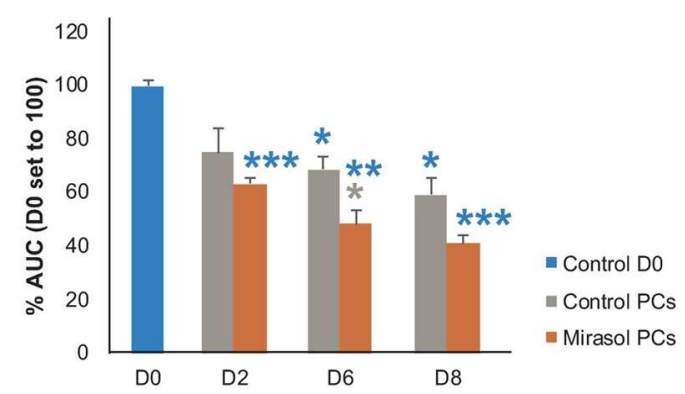

Fig. 1

Figure 1. Flow cytometry-based PLT aggregation assay. Aggregation of PLTs from Control and Mirasol PRT

PCs at day 2, day 6, and day 8 of storage upon addition of the agonists PMA, collagen, Convulxin, and Ristocetin over an incubation period of 5 minutes is depicted. Aggregation was measured by flow cytometry as percentage of double-colored events through a time-series course, and the Area Under the Curve (AUC) was calculated. Mean and SEM of three independent experiments are shown. D0 are fresh platelets used as reference. $* p<0.05, * * p<0.01, * * * p<0.001$. When the asterisk is blue, the comparison was made with D0 fresh reference platelets. When the asterisk is grey, comparison has been made with the day Control platelet samples.

$138 \times 98 \mathrm{~mm}(300 \times 300 \mathrm{DPI})$ 
A Platelet Concentrate Preparation

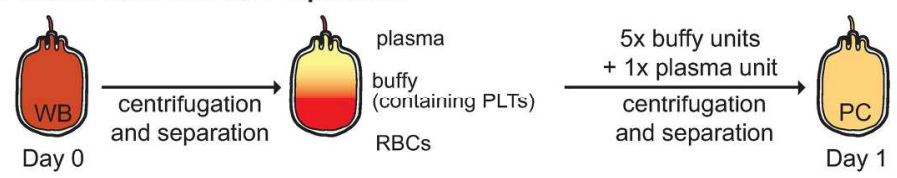

B Experimental Setup

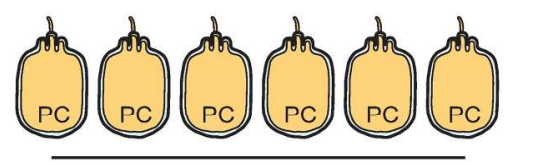

6x PCs are pooled and split again -

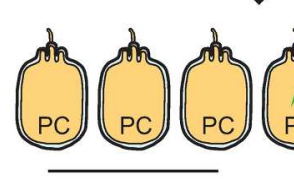

$3 x P C s$ are

left untreated

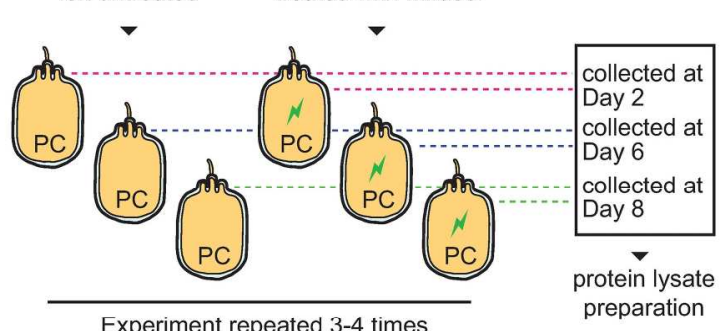

Experiment repeated 3-4 times
Day 1

collected at Day 1

Day 1

C Fresh Platelet Sample Preparation

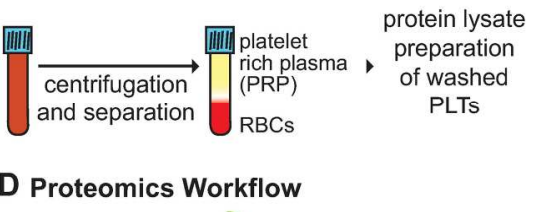

\section{Proteomics Workflow}

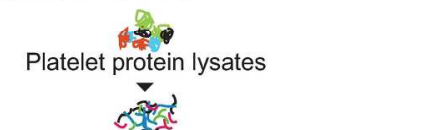

In-solution trypsin digestion

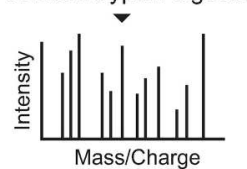

LC-MS/MS analysis

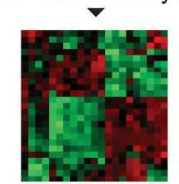

MaxQuant/Perseus Analysis Label-Free Quantification

\section{E Principal Component Analysis}

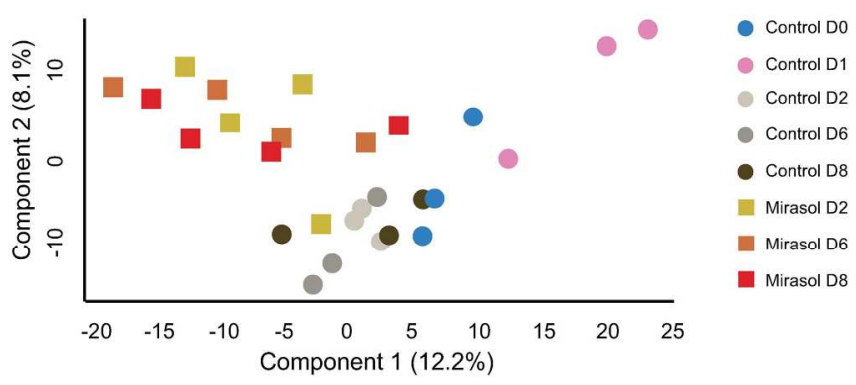

Fig. 2

Figure 2. Schematic overview of the sample preparation and proteomics workflow. (A) Platelet concentrate (PC) preparation is done using a buffy coat-based differential centrifugation method. 1 PC unit is made of 5 units of donated whole blood (WB) +1 unit plasma. RBCs, red blood cells; PLTs, platelets. (B) Experimental setup for Mirasol PRT and Control PC sampling. On each experiment, 6 PCs were pooled together and divided again into 6 units. From these, 3 units were Mirasol PRT treated and the other 3 were left untreated (Control PCs). Samples for platelet function and protein lysate preparation were collected from individual units at day 1, 2, 6 and 8. (C) Fresh platelets were isolated by differential centrifugation, and were subsequently used for functional analysis and protein lysate preparation. (D) Equal amounts ( $5 \mu \mathrm{g}$ protein) from platelet lysate samples were subject to trypsin digestion (in-solution digestion), followed by Orbitrap Fusion $^{\mathrm{TM}}$ Tribrid $^{\mathrm{TM}} \mathrm{MS}$ analysis. Proteins were identified using MaxQuant version 1.5.5.1

(http://www.maxquant.org) coupled to Andromeda search engine. Subsequent label-free quantification bioinformatic analysis was performed in Perseus software version 1.5.5.3 (http://www.perseusframework.org). (E) Principal Component Analysis of all files included in the study. D0 
1

2

3

4

5

6

7

8

9

10

11

12

13

14

15

16

17

18

19

20

21

22

23

24

25

26

27

28

29

30

31

32

33

34

35

36

37

38

39

40

41

42

43

44

45

46

47

48

49

50

51

52

53

54

55

56

57

58

59

60

are fresh platelets.

$207 \times 251 \mathrm{~mm}(300 \times 300 \mathrm{DPI})$

URL: http://mc.manuscriptcentral.com/cpla E-mail: P.Harrison.1@bham.ac.uk 
B Pearson Correlation

Correlation

High

Low

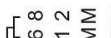

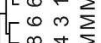

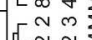

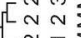

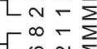

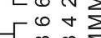

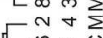

ש0.-

$\begin{array}{llll}0 & \infty & 0 \\ \infty & \infty & 0 \\ 0 & 0 & 0 \\ 0 & 0\end{array}$

$\left[\begin{array}{ccc}\infty & \infty & 0 \\ -\infty & \sim & 0 \\ 0 & \sim & 0 \\ 0 & 0\end{array}\right.$

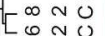

$\begin{array}{rl}1 & -20 \\ 0 & 0\end{array}$

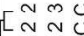

$\begin{array}{rl}n & -0 \\ n & n\end{array}$

Fig. 3
A Principal Component Analysis

C Unsupervised Hierarchical Clustering Intensity
High High

Low

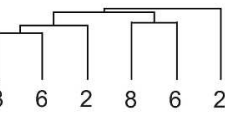

$\begin{array}{lllllll}\text { Day } & 8 & 6 & 2 & 8 & 6 & 2\end{array}$ Treatment M M M C C C
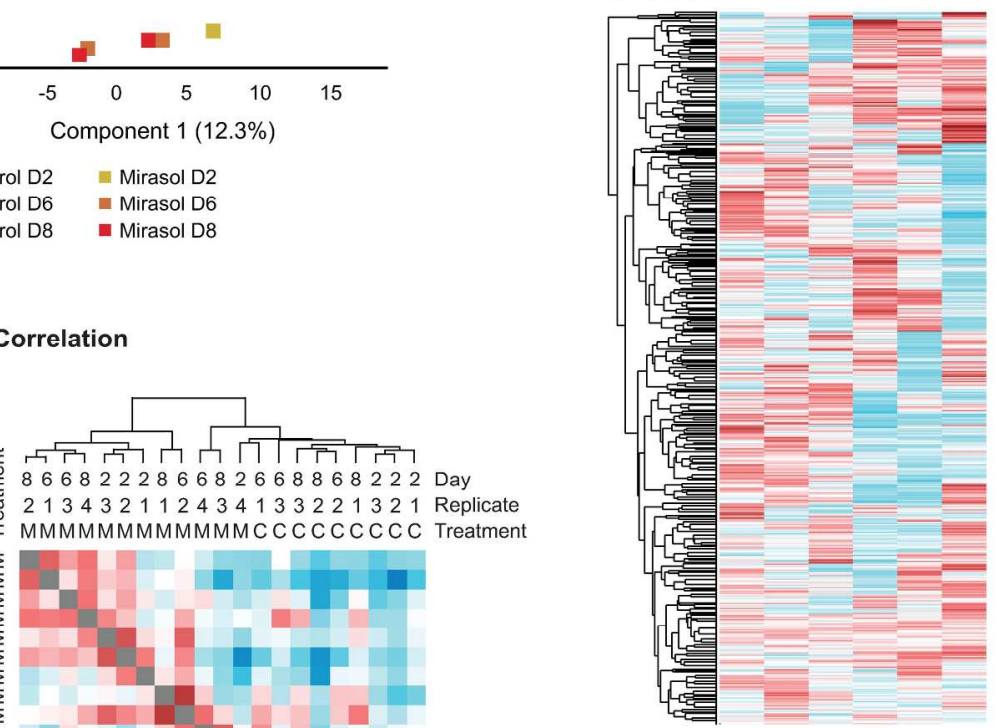

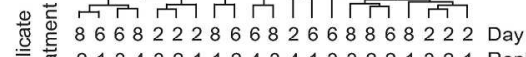

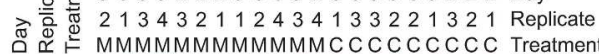

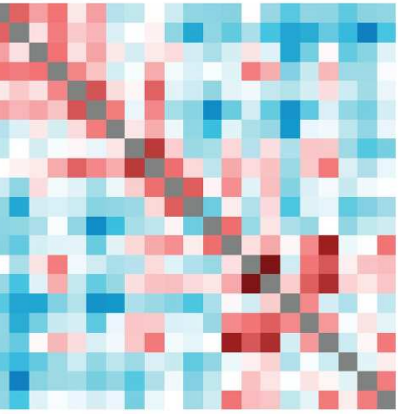

D Pearson Correlation (Averaged Replicates)
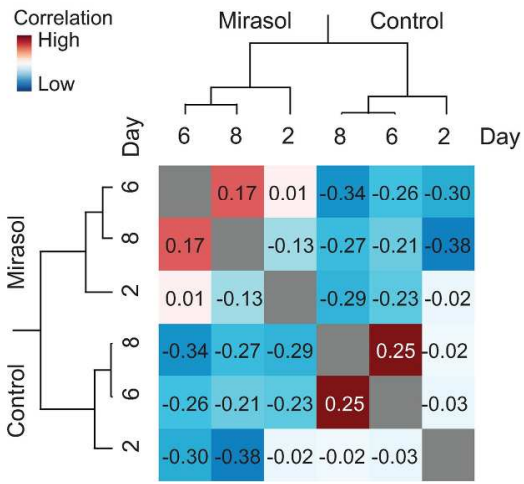

Figure 3. Proteome comparison Mirasol PRT and Control PCs during storage. (A) Principal Component Analysis of Mirasol PRT and Control PCs at days 2, 6 and 8. (B) Matrix representation of Pearson correlation values of the proteome of Mirasol PRT and Control PC platelet lysate replicates through storage. (C) Twoway unsupervised hierarchical clustering of the median protein expression values of the proteome of Control and Mirasol PRT platelet lysate samples at day 2, 6 and 8 of storage. (D) Matrix representation of Pearson correlation values of the proteomes of each averaged stored Control and Mirasol PRT platelet sample-group against the others.

$215 \times 279 \mathrm{~mm}(300 \times 300 \mathrm{DPI})$ 


\section{A Unsupervised Hierarchical Clustering}

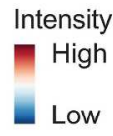

Low

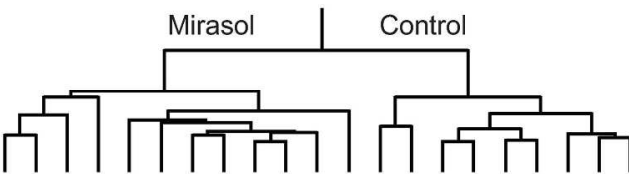

Day $2 \begin{array}{lllllllllllllllllllll}2 & 2 & 2 & 8 & 8 & 6 & 8 & 6 & 6 & 8 & 6 & 8 & 8 & 6 & 6 & 8 & 6 & 2 & 2 & 2\end{array}$ Replicate $\begin{array}{lllllllllllllllllllll} & 1 & 2 & 4 & 4 & 3 & 2 & 1 & 4 & 3 & 2 & 1 & 3 & 2 & 3 & 2 & 1 & 1 & 2 & 3 & 1\end{array}$

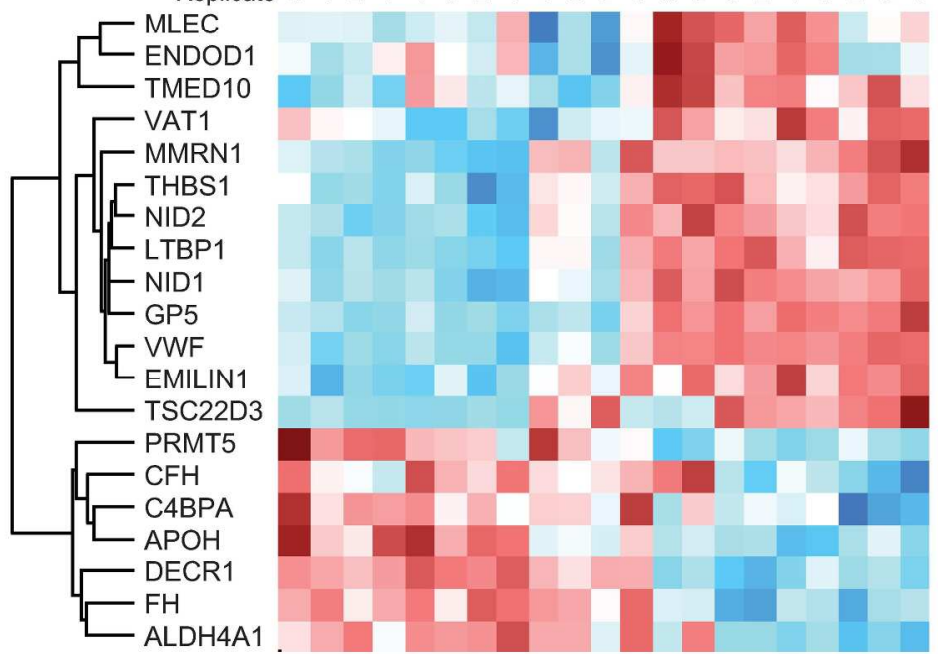

\section{B Cellular Component}

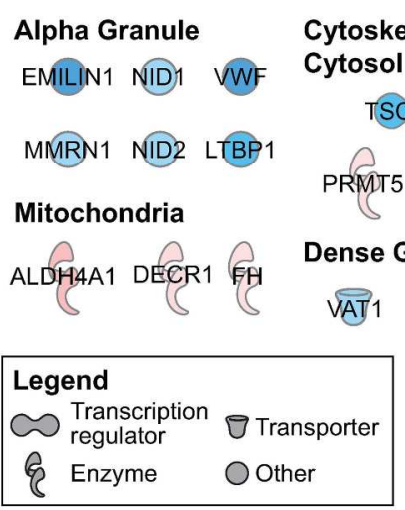

\author{
Plasma Membrane \\ and Extracellular \\ C4BPA CFH THBS1 \\ Plasma Membrane \\ and ER \\ GP5 APOH \\ MLEC TMED10
}

Fig. 4

Figure 4. Proteins differentially expressed in Mirasol PCs are minimal, and locate to alpha-granules. (A) Twoway unsupervised hierarchical clustering of the normalized expression values of all 20 significant proteins was performed. Control and Mirasol PRT PC sample replicates were clustered. (B) Graphical representation of the DE proteins indicating their assigned cellular compartment. Most of the downregulated DE proteins are located to alpha-granules, while most of the upregulated DE proteins are located to mitochondria and plasma membrane.

$182 \times 295 \mathrm{~mm}(300 \times 300 \mathrm{DPI})$ 


\section{A Western Blot: Platelet Lysates}

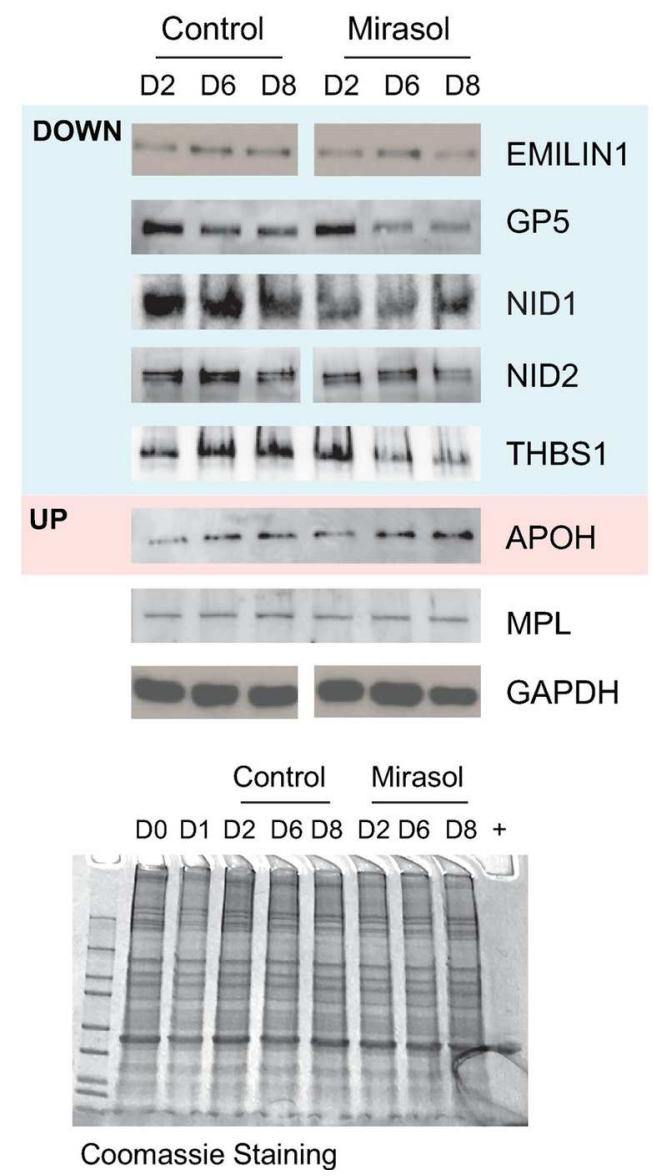

Fig. 5

\section{B Western Blot: PCs Supernatants}
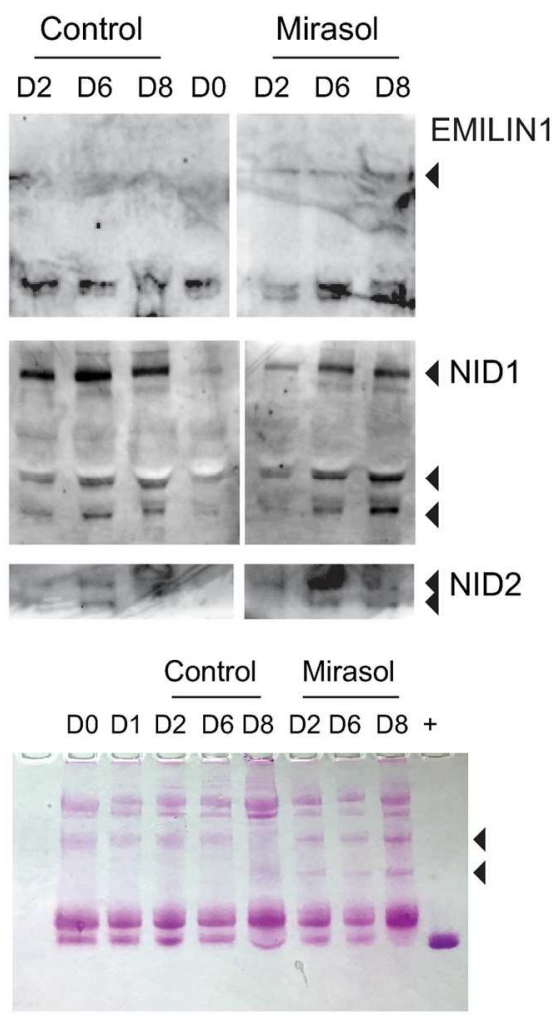

Glycoprotein Staining

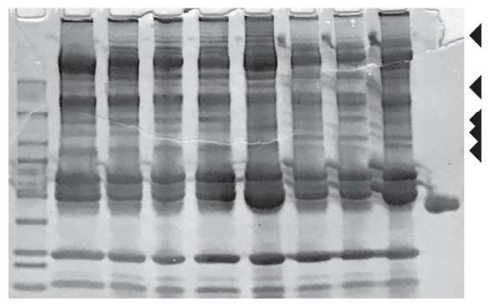

Coomassie Staining

Figure 5. Western blot analysis of DE proteins in platelet lysates and PC supernatants. (A) Western blot analysis of platelet lysates: the figure panel displays representative Western blot analyses of EMILIN1, GP5, NID1, NID2 and THBS1 (downregulated, within blue box); APOH (upregulated, within pink box); and MPL and GAPDH expression in Control and Mirasol PRT platelets through storage. The lysate volume corresponding to the same number of platelets was loaded. Coomassie staining is provided as loading control, including lysate from fresh platelets (D0). (B) Western blot analysis of PC supernatants: the figure panel displays representative Western blot analyses of EMILIN1, NID1, NID2 and THBS1 in Control and

Mirasol PRT PC supernatants through storage. D0 in this panel is plasma from fresh blood samples. The same volume of IgG/albumin depleted supernatants was loaded. Glycoprotein and Coomassie stainings are provided. 
A Principal Component Analysis

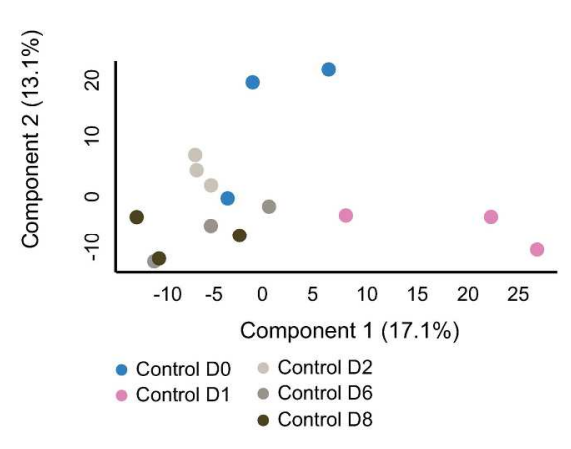

B Pearson Correlation

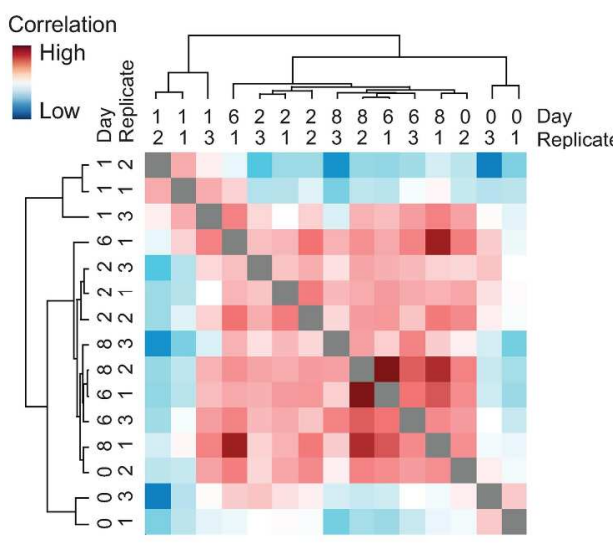

Fig. 6

\section{Unsupervised Hierarchical Clustering}

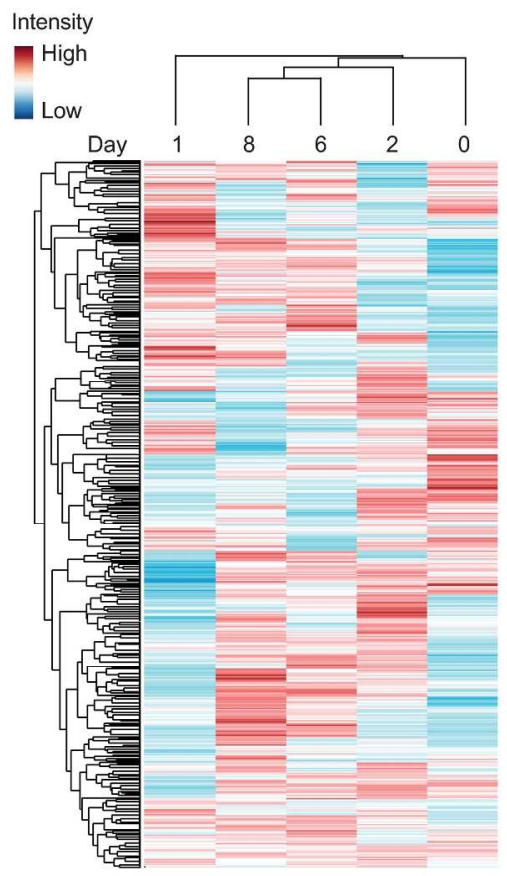

D Pearson Correlation (Averaged Replicates)

Correlation

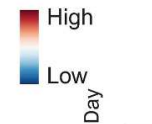

Figure 6. Proteome comparison of fresh (D0) platelets and Control PCs during storage. (A) Principal Component Analysis of Control PCs at days 1, 2, 6 and 8. D0 are fresh platelets. (B) Matrix representation of Pearson correlation values of the proteome of stored Control and fresh (D0) platelet lysate replicates. (C) Two-way unsupervised hierarchical clustering of the median protein expression values of the proteome of fresh (D0) and stored platelet samples at day 1, 2, 6 and 8 through storage. (D) Matrix representation of Pearson correlation values of the proteomes of each averaged stored Control and fresh platelet samplegroup against the others.

$$
221 \times 287 \mathrm{~mm}(300 \times 300 \mathrm{DPI})
$$


Table 1: Significant Protein Changes after Multiple Sample Test of Control vs Mirasol PC Lysates through Storage Indicated are the Log2 LFQ fold ratios Mirasol vs Control at days 2, 6 and 8 of Storage,

Multiple Sample test ANOVA FDR, and regulation of expression Mirasol vs Control.

$L F Q=$ Label Free Quantification; FDR = false discovery rate; $C=$ Control; $M=$ Mirasol, UP = upregulated; $D O W N=$ downregulated.

\begin{tabular}{|c|c|c|c|c|c|c|}
\hline \multirow[b]{2}{*}{ Gene Symbol } & \multirow[b]{2}{*}{ Description } & \multicolumn{4}{|c|}{ Log2 LFQ Ratio and ANOVA } & \multirow[b]{2}{*}{ Regulation } \\
\hline & & M vs C D2 & M vs C D6 & M vs C D8 & FDR & \\
\hline ALDH4A1 & Delta-1-pyrroline-5-carboxylate dehydrogenase, mitochondrial & 1,0676 & 0,7687 & 0,6679 & 0,0378 & UP \\
\hline $\mathrm{APOH}$ & Beta-2-glycoprotein 1 & 1,0804 & 1,2460 & 0,7997 & 0,0052 & UP \\
\hline C4BPA & C4b-binding protein alpha chain & 1,7272 & 2,2756 & 3,0584 & 0,0001 & UP \\
\hline $\mathrm{CFH}$ & Complement factor $\mathrm{H}$ & 0,3387 & 1,0191 & 1,0566 & 0,0197 & UP \\
\hline DECR1 & 2,4-dienoyl-CoA reductase, mitochondrial & 0,7780 & 0,3552 & 0,2126 & 0,0220 & UP \\
\hline $\mathrm{FH}$ & Fumarate hydratase, mitochondrial & 0,3816 & 0,2109 & 0,0028 & 0,0267 & UP \\
\hline PRMT5 & Protein arginine $\mathrm{N}$-methyltransferase 5 & 0,1895 & 0,3116 & 0,2448 & 0,0201 & UP \\
\hline EMILIN1 & EMILIN-1 & $-1,0460$ & $-1,4378$ & $-1,5258$ & 0,0009 & DOWN \\
\hline ENDOD1 & Endonuclease domain-containing 1 protein & $-0,1827$ & $-0,4005$ & $-0,4226$ & 0,0178 & DOWN \\
\hline GP5 & Platelet glycoprotein V & $-0,5377$ & $-0,8690$ & $-1,0820$ & 0,0052 & DOWN \\
\hline LTBP1 & Latent-transforming growth factor beta-binding protein 1 & $-0,4373$ & $-0,7985$ & $-1,0173$ & 0,0027 & DOWN \\
\hline MLEC & Malectin & $-0,5541$ & $-0,6484$ & $-0,7697$ & 0,0361 & DOWN \\
\hline MMRN1 & Multimerin-1 & $-0,2862$ & $-0,4265$ & $-0,5825$ & 0,0020 & DOWN \\
\hline NID1 & Nidogen-1 & $-0,3172$ & $-0,5334$ & $-0,7472$ & 0,0305 & DOWN \\
\hline NID2 & Nidogen-2 & $-0,3802$ & $-0,5128$ & $-0,5977$ & 0,0023 & DOWN \\
\hline TMED10 & Transmembrane emp24 domain-containing protein 10 & $-0,7426$ & $-0,7527$ & $-0,6430$ & 0,0322 & DOWN \\
\hline TSC22D3 & TSC22 domain family protein 3 & $-0,4801$ & $-0,9435$ & $-0,4715$ & 0,0170 & DOWN \\
\hline THBS1 & Thrombospondin-1 & $-0,4058$ & $-0,6140$ & $-0,7743$ & 0,0025 & DOWN \\
\hline VAT1 & Synaptic vesicle membrane protein VAT- 1 homolog & $-0,2632$ & $-0,5265$ & $-0,6539$ & 0,0203 & DOWN \\
\hline WWF & von Willebrand factor; von Willebrand antigen 2 & $-1,0355$ & $-1,0825$ & $-1,1528$ & 0,0019 & DOWN \\
\hline
\end{tabular}




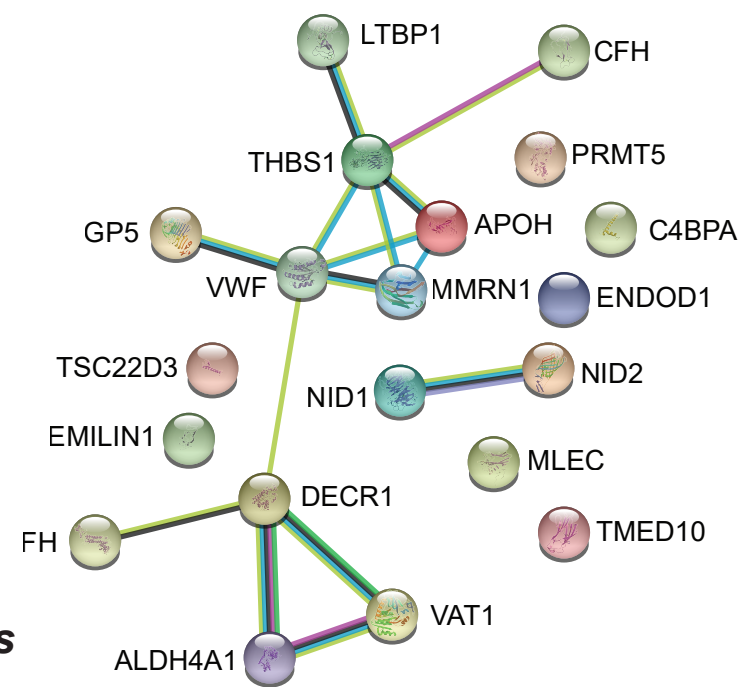

\section{DE proteins}

ALDH4A

Biological Process

\begin{tabular}{llcc}
\hline \#pathw ay ID & pathw ay description & Gene Nr. & FDR \\
\hline GO.0072376 & protein activation cascade & 5 & $5.70 \mathrm{E}-05$ \\
GO.0007597 & blood coagulation, intrinsic pathw ay & 3 & 0.00229 \\
GO.0030198 & extracellular matrix organization & 6 & 0.00229 \\
GO.0072378 & blood coagulation, fibrin clot formation & 3 & 0.005 \\
GO.0031589 & cell-substrate adhesion & 4 & 0.0275 \\
GO.1903034 & regulation of response to w ounding & 5 & 0.0357 \\
\hline & & & \\
Molecular Function & Gene Nr. & FDR \\
\hline \#pathw ay ID & pathw ay description & 5 & $1.57 \mathrm{E}-05$ \\
GO.0001948 & glycoprotein binding & 4 & 0.000165 \\
GO.0005518 & collagen binding & 3 & 0.000609 \\
GO.0043394 & proteoglycan binding & &
\end{tabular}

\section{Cellular Component}

\section{\#pathway ID pathway description}

GO.0005576 extracellular region

Go.0044421 extracellular region part

G0.0031012 extracellular matrix

Go.0031988 membrane-bounded vesicle

Go.0070062 extracellular exosome

GO.0005578 proteinaceous extracellular matrix

GO.0031093 platelet alpha granule lumen

Go.0031091 platelet alpha granule

GO.0030141 secretory granule

G0.0044420 extracellular matrix component

\begin{tabular}{cc} 
Gene Nr. & FDR \\
\hline 15 & 0.000177 \\
14 & 0.000177 \\
6 & 0.000206 \\
13 & 0.000206 \\
12 & 0.000206 \\
5 & 0.00199 \\
3 & 0.002 \\
3 & 0.0036 \\
4 & 0.0162 \\
3 & 0.0182 \\
\hline
\end{tabular}

KEGG

\begin{tabular}{llcc}
\hline \#pathw ay ID & pathw ay des cription & Gene Nr. & FDR \\
\hline 4512 & ECM-receptor interaction & 3 & 0.0108 \\
4610 & Complement and coagulation cascades & 3 & 0.0108 \\
\hline
\end{tabular}

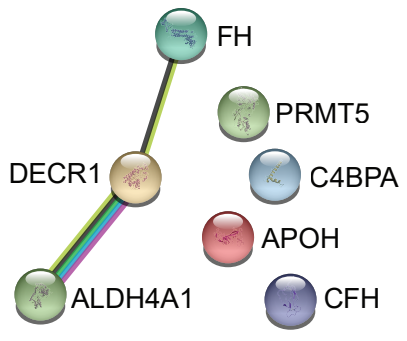

Biological Process
Downregulated DE proteins

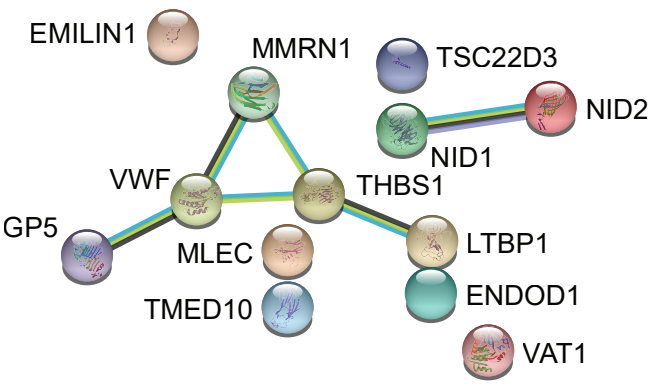

Biological Process

\begin{tabular}{llcc}
\hline \#pathw ay ID & pathw ay description & Gene Nr. & FDR \\
\hline G0.0030198 & extracellular matrix organization & 6 & 0.000224 \\
G0.0031589 & cell-substrate adhesion & 4 & 0.00844 \\
G0.0030168 & platelet activation & 4 & 0.0336 \\
GO.0002576 & platelet degranulation & 3 & 0.0403 \\
G0.0006887 & exocytosis & 4 & 0.0403 \\
GO.0071711 & basement membrane organization & 2 & 0.0403 \\
\hline
\end{tabular}

Molecular Function

\begin{tabular}{llcc}
\hline \#pathw ay ID & pathw ay description & Gene Nr. & FDR \\
\hline G0.0005518 & collagen binding & 4 & $4.91 \mathrm{E}-05$ \\
G0.0001948 & glycoprotein binding & 3 & 0.0168 \\
G0.0043236 & laminin binding & 2 & 0.0415 \\
G0.0043394 & proteoglycan binding & 2 & 0.0415 \\
GO.0050431 & transforming grow th factor beta binding & 2 & 0.0415 \\
\hline
\end{tabular}

Cellular Component

\begin{tabular}{llcl}
\hline \#pathway ID & pathway description & Gene Nr. & FDR \\
\hline G0.0031012 & extracellular matrix & 6 & $6.52 E-05$ \\
G0.0005578 & proteinaceous extracellular matrix & 5 & 0.000903 \\
G0.0031093 & platelet alpha granule lumen & 3 & 0.00186 \\
G0.0031091 & platelet alpha granule & 3 & 0.00255 \\
G0.0005576 & extracellular region & 10 & 0.00379 \\
G0.0030141 & secretory granule & 4 & 0.00413 \\
G0.0031988 & membrane-bounded vesicle & 9 & 0.00413 \\
G0.0044420 & extracellular matrix component & 3 & 0.00521 \\
G0.0044421 & extracellular region part & 9 & 0.00521 \\
G0.0070062 & extracellular exosome & 8 & 0.00521 \\
G0.0044433 & cytoplasmic vesicle part & 4 & 0.0195 \\
\hline
\end{tabular}

KEGG

\begin{tabular}{llcc}
\hline \#pathw ay ID & pathway description & Gene Nr. & FDR \\
\hline 4512 & ECM-receptor interaction & 3 & 0.00551 \\
\hline
\end{tabular}

\begin{tabular}{llcc}
\hline \#pathway ID & pathway description & Gene Nr. & FDR \\
\hline GO.0072376 & protein activation cascade & 3 & 0.0146 \\
\hline
\end{tabular}




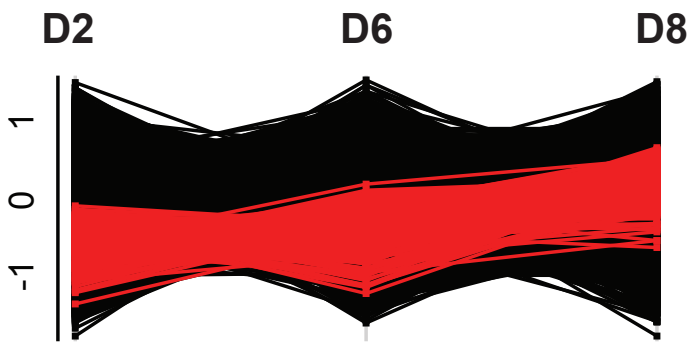

Cluster 1:

vesicle mediated transport, catabolim, cellular stress,

immune response

CYFIP2, NBEAL2, PSMA1

MYLK, ALDH4A1

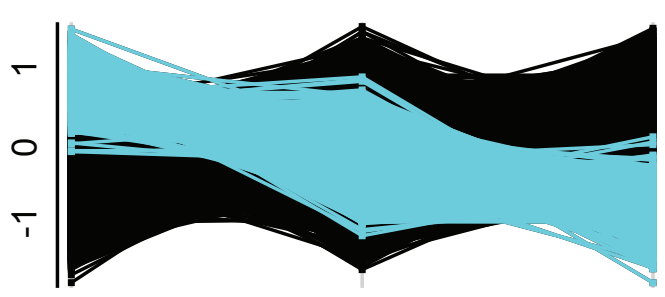

Cluster 4:

metabolism, vesicle mediated transport, immune response

\section{GP5, THBS1, MMRN1, NID2 \\ EMILIN1, TSC22D3, C4BP4}

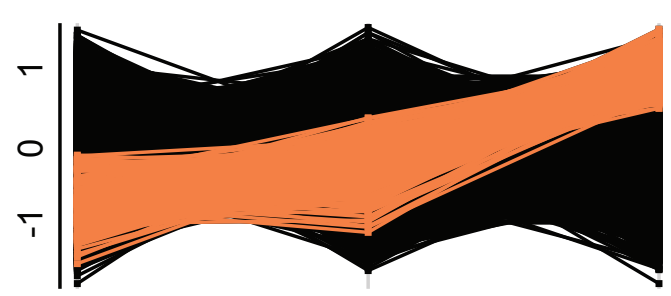

Cluster 7:

oxidation-reduction process, metabolism, intracellular transport

G6PD, GP1BB, ITGA2,

ITGB1, TMED10, FH

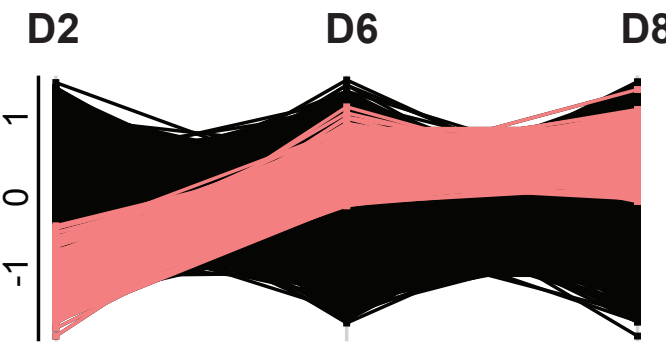

\section{Cluster 2:}

hemostasis, blood coagulation, platelet activation, wound healing

\section{FLNA, ITGB3, SELP, SYK, GP9} ITGB3, MLEC, ENDOD1, DECR1

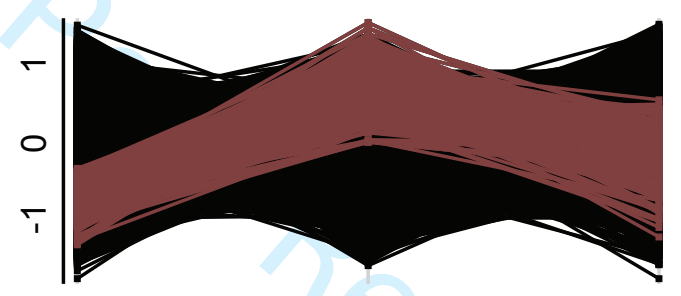

\section{Cluster 5:}

cytoskeleton, protein transport, vesicle mediated transport

ALOX12, CLIP1, MYH9, STXBP3 BTK, ITGA2B, LTBP1

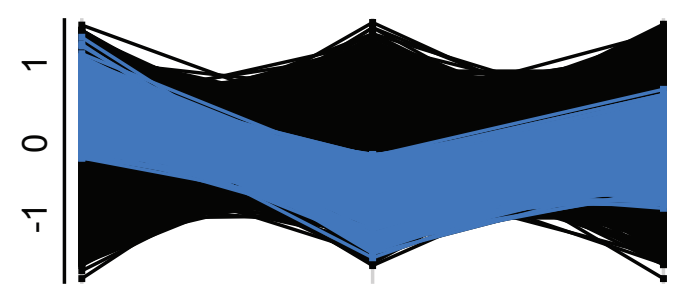

Cluster 8:

catabolic process, vesicle mediated transport

ARFGEF1, FUT8, PPIA, STXBP5 GP1BA, CLEC1B

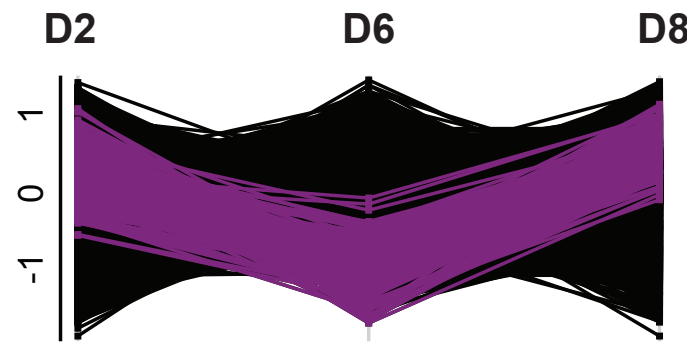

Cluster 3:

receptor signalling, signal transduction, platelet activation

ARHGEF7, MPL VAT1, NID1, APOH

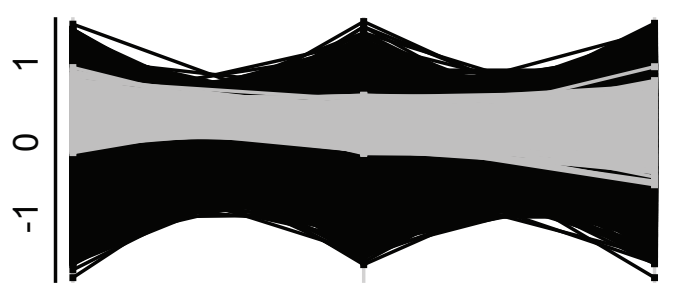

\section{Cluster 6:}

protein transport, protein localization

\section{ANXA1, ARFGAP1, CLASP2 CFH}

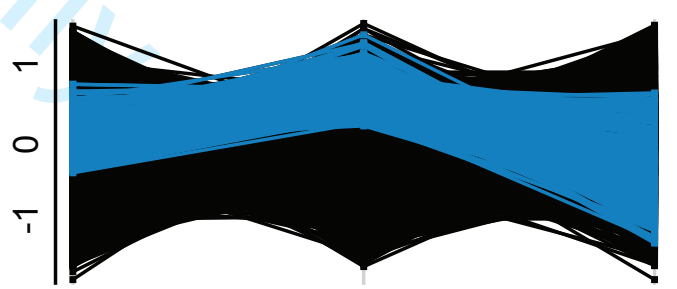

\section{Cluster 9:}

vesicle mediated transport, establishment of protein localization

COG1, DAB2, EXOC3, IPO7 FCGR2A 


\section{SUPPORTING FILES LEGENDS}

Figure S1: STRING analysis of differentially expressed (DE) proteins after Multiple Sample Test Analysis of Mirasol PRT vs Control PCs. Protein-protein functional interactions and GO-Term enrichment are depicted for the total of DE proteins, and separately for down- and upregulated DE proteins (Table S4).

Figure S2. Dynamics of platelet protein changes through storage. Dynamics of protein changes were analyzed by K-means using Perseus software. Time-course kinetics of significant proteins in 9 different clusters is shown. On the X-axis, time points indicate Day 2, Day 6 and Day 8 of platelet storage while on the Y-axis, the respective expression values of proteins in the cluster are depicted. The black lines represent the average expression of all proteins while coloured lines are the expression (LFQ intensity value) of the clustered proteins. Below, relevant enriched Biological Process GO-Terms are shown. Proteins DE in Mirasol vs Control PCs are also indicated (blue, downregulated and red, upregulated). Other proteins of interest within the cluster are indicated.

Table S1: Proteomic Analysis of Control vs Mirasol PC Lysates through Storage. Indicated are the Average Log2 LFQ of Control and Mirasol PCs samples at Days 2, 6 and 8 of Storage, Log2 LFQ fold ratios Mirasol vs Control at different Days, FDR ( $t$ test), Multiple Sample test ANOVA significant entries and corresponding FDR, and the individual Log2 LFQ values of individual Control and Mirasol Samples. LFQ = Label Free Quantification; $\mathrm{FDR}=$ false discovery rate $\mathrm{C}=$ Control; $\mathrm{M}=$ Mirasol, $\mathrm{D}=$ Day.

Table S2: Gene Ontology (GO) Term (GO-Term) enrichment analysis of significantly differentially expressed (DE) proteins identified after Multiple Sample test comparing Control and Mirasol PRT PCs through storage. From the 20 significant DE proteins, 
STRING-based GO-Term enrichment analysis was performed on the total, down-and upregulated datasets. All identified Terms are depicted, including Biological Process, Biological Function, Cellular component and KEGG pathway.

\section{Table S3: Proteomic Analysis of Fresh Platelets and Control PC Lysates through} Storage. Indicated are the Average Log2 LFQ of Fresh Platelets and Control PCs samples at Days 1, 2, 6 and 8 of Storage, Log2 LFQ fold ratios Control at different Days vs Fresh or Day 1 Platelets, FDR (T test), Multiple Sample test ANOVA FDR, and the individual Log2 LFQ values of Fresh Platelets and Control PC Samples. LFQ = Label Free Quantification; FDR = false discovery rate; $\mathrm{C}=$ Control; $\mathrm{D}=$ Day; $\mathrm{D} 0=$ Fresh Platelets.

Table S4: K-means cluster analysis of Control PCs through storage. First datasheet contains a table indicating the proteins enlisted on each identified cluster (See Fig. S2). Second datasheet contains significant Biological Processes (maximum 15) after STRINGbased GO-Term enrichment performed separately with the protein list from each cluster. 